\title{
New Delhi metallo- $\beta$-lactamase-1: local acquisition in Ontario, Canada, and challenges in detection
}

\author{
Julianne V. Kus PhD, Manal Tadros MBBS PhD, Andrew Simor MD, Donald E. Low MD, \\ Allison J. McGeer MSc MD, Barbara M. Willey ART, Cindy Larocque MLT, Karen Pike MLT, \\ Iris-Ann Edwards MLT, Helen Dedier MLT, Roberto Melano PhD, David A. Boyd MSc, Michael R. Mulvey PhD, \\ Lisa Louie ART, Christopher Okeahialam MSc CIC, Mark Bayley MD, Cynthia Whitehead MScCH MD, \\ Denyse Richardson MEd MD, Lesley Carr MD, Fatema Jinnah MBBS MSc, Susan M. Poutanen MD MPH
}

See related commentary by Walsh on page 1240 and at www.cmaj.ca/lookup/doi/10.1503/cmaj.111100.

\section{- ABSTRACT}

New Delhi metallo- $\beta$-lactamase-1 (NDM-1) is a recently identified metallo- $\beta$-lactamase that confers resistance to carbapenems and all other $\beta$-lactam antibiotics, with the exception of aztreonam. NDM-1 is also associated with resistance to many other classes of antibiotics. The enzyme was first identified in organisms isolated from a patient in Sweden who had previously received medical treatment in India, but it is now recognized as endemic throughout India and Pakistan and has spread worldwide. The gene encoding NDM-1 has been found predominantly in Escherichia coli and Klebsiella pneumoniae. We describe the isolation NDM-1-producing organisms from two patients in Toronto, Ontario. To the best of our knowledge, this is the first report of an organism producing NDM-1 that was locally acquired in Canada. We also discuss the evidence that NDM-1 can affect bacterial species other than $E$. coli and $K$. pneumoniae, the limited options for treatment and the difficulty laboratories face in detecting organisms that produce NDM-1.
$\mathrm{N}$ ew Delhi metallo- $\beta$-lactamase-1 (NDM1 ) is a metallo- $\beta$-lactamase that confers resistance to carbapenems and all other $\beta$ lactam antibiotics, with the exception of aztreonam. It is predominantly found in the Enterobacteriaeceae. It was first identified in Escherichia coli and Klebsiella pneumoniae isolated from a patient in Sweden who had previously received medical treatment in India. It is now recognized as endemic throughout India and Pakistan and has spread worldwide due to travel, "medical tourism" and the ability of the genetic element encoding the enzyme to transfer between bacteria. ${ }^{1-3}$ Three reports of organisms producing NDM-1 in Canada have been published to date. In each instance, the organisms were isolated from the urinary tracts of patients who had recently been admitted to hospitals in India. Two of the isolates were strains of $K$. pneumoniae and one was a strain of E. coli. ${ }^{46}$ Additional reports of isolation of organisms producing NDM-1 from patients in Canada have been presented in the lay press.

Organisms that produce NDM-1 have been associated with resistance to classes of antibiotics other than the $\beta$-lactams, thus severely limiting options for treatment. ${ }^{2}$ Infection control guidance regarding the management of colonization by or infection with organisms that produce carbapenemases, such as NDM-1, have recently been published by Canadian and European authorities. ${ }^{7-9}$ An essential component of these recommendations is the rapid and accurate identification of the organisms in a clinical microbiology laboratory. The Clinical Laboratory Standards Institute (CLSI) and the United States Centers for Disease Control and Prevention (CDC) recommend screening for the production of carbapenemase using the Modified Hodge Test. ${ }^{10,11}$ If the result of that test is positive, then the presence and type of carbapenemase can be confirmed by polymerase chain reaction. ${ }^{4}$

Herein, we summarize two additional instances in which organisms producing NDM-1 were isolated from patients in Canada and the first where the organism appears to have been acquired in Canada.
Competing interests: Allison McGeer is a member of the board for Bayer and Pfizer and has received grants and speaker fees from same. No competing interests were declared by the other authors.

This article has been peer reviewed.

Correspondence to: Dr. Susan M. Poutanen, spoutanen@mtsinai.on.ca

CMAJ 2011. DOI:10.1503 /cmaj.110477 


\section{Case one}

An 86-year-old man was admitted to a tertiarycare teaching hospital in Toronto after having had a stroke. He was later discharged to a nearby inpatient facility for rehabilitation. The patient had not travelled outside of southwestern Ontario for at least the past 10 years. His medical history included previous strokes, type 2 diabetes mellitus, hypertension, atrial fibrillation, colon cancer, prostate cancer and colonization with methicillin-resistant Staphylococcus aureus (MRSA). At both the hospital and the rehabilitation facility, contact precautions were taken for MRSA colonization. The patient's stay at the rehabilitation facility was complicated by acute cholecystitis, and he was transferred back to the hospital where he was given treatment with intra- venous ceftriaxone and metronidazole. A culture of the patient's urine grew a multidrug-resistant strain of Morganella morganii that showed intermediate susceptibility to ertapenem and susceptibility to meropenem based on testing with the Vitek-2 automated susceptibility testing system (bioMérieux, France) using the interpretive breakpoints stipulated in the CLSI Performance Standards for Antimicrobial Susceptibility Testing; Twentieth Informational Supplement (CLSI document number M100-S20, issued in January 2010). ${ }^{12}$ Carbapenemases were not detected by the Modified Hodge Test. Because the patient had no urinary symptoms, the bacteriuria was not treated. The patient's condition improved after several days, and he was again discharged to the rehabilitation facility.

On readmission to the rehabilitation facility,

Table 1: Results of antimicrobial susceptibility tests of two b/a ${ }_{\mathrm{NDM}-1}$ isolates described in this report

\begin{tabular}{|c|c|c|c|c|}
\hline \multirow[b]{3}{*}{ Antimicrobial agent } & \multicolumn{4}{|c|}{ Isolate } \\
\hline & \multicolumn{2}{|c|}{$\begin{array}{c}\text { bla }_{\mathrm{NDM}-1}-\text { positive Morganella } \\
\text { morganii* }\end{array}$} & \multicolumn{2}{|c|}{ 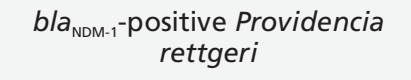 } \\
\hline & $\mathrm{MIC}, \mathrm{mg} / \mathrm{L} \dagger$ & Interpretation§ & $\mathrm{MIC}, \mathrm{mg} / \mathrm{L} \dagger$ & Interpretation§ \\
\hline Ertapenem & 4 & $\mathrm{R}$ & 8 & $\mathrm{R}$ \\
\hline Imipenem & 16 & $\mathrm{R}$ & $\geq 32$ & $\mathrm{R}$ \\
\hline Meropenem & 8 & $\mathrm{R}$ & $\geq 32$ & $\mathrm{R}$ \\
\hline Doripenem & $\geq 8$ & $\mathrm{R}$ & $\geq 8$ & $\mathrm{R}$ \\
\hline Ampicillin & $\geq 32 \ddagger$ & $\mathrm{R}$ & $\geq 32 \ddagger$ & $\mathrm{R}$ \\
\hline Amoxicillin/clavulinic acid & $\geq 32 / 16 \ddagger$ & $\mathrm{R}$ & $\geq 32 / 16 \ddagger$ & $\mathrm{R}$ \\
\hline Cefazolin & $\geq 64 \ddagger$ & $\mathrm{R}$ & $\geq 64 \ddagger$ & $\mathrm{R}$ \\
\hline Ceftriaxone & $\geq 64 \ddagger$ & $\mathrm{R}$ & $32 \ddagger$ & $\mathrm{R}$ \\
\hline Ceftazidime & $\geq 64$ & $\mathrm{R}$ & $\geq 64$ & $\mathrm{R}$ \\
\hline Cefepime & $\geq 64$ & $\mathrm{R}$ & 32 & $\mathrm{R}$ \\
\hline Amikacin & $\geq 128$ & $\mathrm{R}$ & $\geq 128$ & $\mathrm{R}$ \\
\hline Gentamicin & $\geq 32$ & $\mathrm{R}$ & $\geq 32$ & $\mathrm{R}$ \\
\hline Tobramycin & $\geq 32$ & $\mathrm{R}$ & $\geq 32$ & $\mathrm{R}$ \\
\hline Ciprofloxacin & $\geq 8$ & $\mathrm{R}$ & $\geq 8$ & $\mathrm{R}$ \\
\hline Nitrofurantoin & $128 \ddagger$ & $\mathrm{R}$ & $256 \ddagger$ & $\mathrm{R}$ \\
\hline Tetracycline & $\geq 32$ & $\mathrm{R}$ & $\geq 32$ & $\mathrm{R}$ \\
\hline Chloramphenicol & 32 & $\mathrm{R}$ & 8 & $S$ \\
\hline Colistin & $\geq 16$ & Rף & $\geq 16$ & $\mathrm{R} ף$ \\
\hline Tigecycline & 2 & $S^{* *}$ & 1 & $S^{* *}$ \\
\hline \multicolumn{5}{|c|}{ 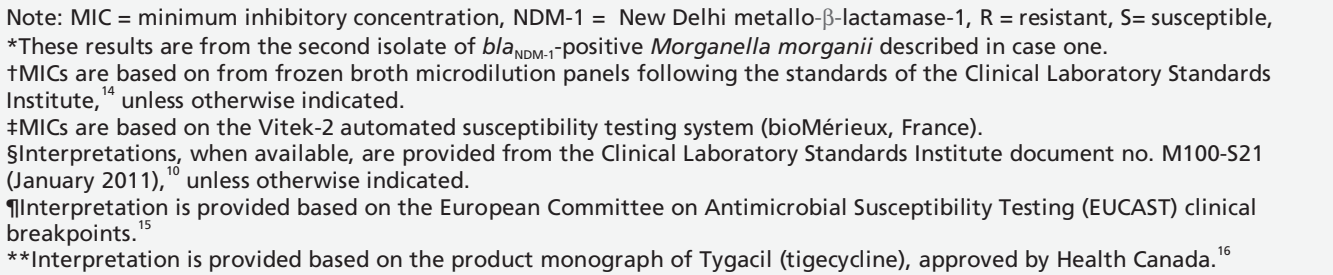 } \\
\hline
\end{tabular}


a culture of the patient's urine again grew a multidrug-resistant strain of $M$. morganii. This time, however, Vitek-2 testing showed that the organism was resistant to ertapenem but susceptible to meropenem. Although a Modified Hodge Test of this isolate also gave a negative result for carbapanemases, as interpreted by both the hospital laboratory and a reference laboratory, ${ }^{13}$ polymerase chain reaction showed that the organism possessed the gene encoding NDM-1 carbapenemase $\left(b l a_{\mathrm{NDM}-1}\right)$. Additional susceptibility testing showed that the organism possessed extensive resistance to drugs (Table 1). Because the patient was asymptomatic, he did not receive treatment.

A second test of the initial M. morganii isolate, which had shown intermediate resistance to ertapenem, showed that it was in fact positive for $b l a_{\mathrm{NDM}-1}$. Pulsed-field gel electrophoresis of the initial isolate and the subsequent ertapenem-resistant isolate showed that they were indistinguishable. ${ }^{17}$ Investigations at both the hospital and the rehabilitation facility failed to identify a source for the organism. None of the patient's family members or friends had travelled to India or nearby regions, and the results of rectal swabs from members of the patient's immediate family were negative for NDM-1-producing organisms. Five months later, urine cultures from the patient continued to grow the NDM-1-producing isolate of $M$. morganii.

\section{Case two}

A 71-year-old female resident of Canada had travelled to India in September 2010 to have the Zamboni Liberation Procedure. ${ }^{18}$ She had multiple sclerosis and a neurogenic bladder requiring a chronic indwelling urinary catheter. She had been admitted to a hospital in New Delhi, India, where she had stayed for 10 days. Her stay in hospital had been uncomplicated; her indwelling urinary catheter had not been changed, and no cultures had been taken.

Three days after being discharged from the hospital in India, upon her return to Canada, the patient became febrile and was seen in the emergency department of a tertiary-care teaching hospital in Toronto. Urine cultures grew Proteus mirabilis that was susceptible to cephalothin. The patient was given treatment with oral cephalexin and her symptoms resolved. Follow-up urine cultures taken two weeks later grew a strain of Providencia rettgeri, which Vitek-2 testing showed to be resistant to ertapenem and meropenem. The isolate was tested directly for carbapenemase genes by polymerase chain reaction, which gave a positive result for $b l a_{\mathrm{NDM}-1}$. Additional susceptibility testing showed that the strain was extensively resistant to drugs (Table 1). The patient had no urinary symptoms and was not given treatment. The Modified Hodge Test completed afterward was interpreted as having a negative result. Three months later, urine cultures from the patient continued to grow the NDM-1-producing isolate of P. rettgeri.

\section{Discussion}

These two scenarios show that local acquisition of an organism producing NDM-1 has already occurred in Ontario, Canada, that $b l a_{\mathrm{NDM}-1}$ has been found in bacterial species other than E. coli and $K$. pneumoniae, that treatment options are limited for infections with NDM-1-producing organisms, and that the detection of NDM-1-producing organisms by a laboratory can be difficult.

The second scenario describes the acquisition of an NDM-1-producing organism after receiving medical care while on the Indian subcontinent, the circumstances under which the majority of people have become colonized or infected so far. However, the patient in the first scenario had not travelled outside of southwestern Ontario for at least 10 years and had no family members or friends with any relevant history of travel. To the best of our knowledge, this is the first reported instance in which an NDM-1-producing organism was locally acquired in Canada.

Both of these scenarios show that $b l a_{\mathrm{NDM}-1}$ can be found in bacteria other than E. coli and K. pneumoniae. Although $b l a_{\mathrm{NDM}-1}$ has most commonly been associated with E. coli and K. pneumoniae, it appears able to readily transfer between common enteric bacteria. ${ }^{2}$ Other organisms in which it has been described include Klebsiella oxytoca, Enterobacter cloacae, Proteus spp., and Citrobacter freundii. ${ }^{2}$ Clinicians and clinical laboratories need to be aware of the diverse bacterial species in which $b l a_{\mathrm{NDM}-1}$ has been detected. For the two scenarios we have described, $M$. morganii and $P$. rettgeri were both initially identified using the Vitek-2 microbial identification system, and the identification was confirmed using an API 20 E (bioMérieux, France).

These two scenarios also underscore the lack of options available for treatment when dealing with NDM-1-producing organisms. ${ }^{2}$ Table 1 shows the extensive patterns of drug resistance of the organisms affecting our two patients. The only treatment option for the first patient was tigecycline, and the only treatment options for the second patient were tigecycline or chloramphenicol. Fortunately, both patients remained asymptomatic and did not need to receive any treatment.

It is important to highlight that screening for, and the subsequent treatment of, asymptomatic bacteriuria is not recommended for older adults 
or patients with catheters. ${ }^{18}$ Indeed, for the patients described here, treatment may have been harmful owing to the risk of adverse effects and the potential for selection of additional antimicrobial resistance.

Given the extensive resistance to drugs and the risk of patient-to-patient transmission associated with NDM-1-producing isolates, the Public Health Agency of Canada recommends that patients identified as being infected with or colonized by an NDM-1-producing organism should be treated in single rooms using contact precautions and enhanced environmental cleaning for the duration of their stay in hospital. Should these patients be readmitted to hospital within 12 months, they should be assumed to be colonized and the same contact precautions should be taken. ${ }^{7}$ If a patient's test results show that an NDM-1-producing organism is first isolated more than 48 hours after admission, the patient's roommates should be screened to identify potential colonization or infection, and the hospital's laboratory records should be reviewed to identify any other NDM-1-producing organisms isolated in the previous 6 to 12 months. If two or more patients within an organization are infected or colonized, active surveillance cultures are recommended for all people who may have had contact with the index patients. European guidelines recommend even more precautionary approaches to protecting patients admitted to hospital and suggest that all hospitals should have preparedness plans in place. ${ }^{8,9}$

Finally, both patients illustrate the challenges of detecting organisms that produce NDM-1. Relying on old CLSI interpretive standards without supplemental tests, such as the Modified Hodge Test, may incorrectly label a carbapenemase-producing organism as carbapenem susceptible, as in case one. To correct this, CLSI developed revised interpretive standards based on updated susceptibility testing data, pharmacokinetic and pharmacodynamic models and data on patient outcomes. ${ }^{10}$ It will take time for commercial laboratory systems to reflect these new standards, and even when these standards are in place, routine susceptibility testing will not identify which carbapenemresistant organisms produce carbapenemase; additional tests will be needed. Since mechanisms of carbapenem resistance other than the production of carbapenemase do not have the same implications for infection control, the Modified Hodge Test is still recommended for this purpose. ${ }^{10,11}$

For both of the patients described in this report, the results of the Modified Hodge Test were interpreted as being negative (Appendix 1, available at www.cmaj.ca/lookup/suppl /doi:10-1503/cmaj.110477/-/DC1). We have since observed false-negative results with other NDM-1-producing organisms, ${ }^{20}$ and similar results have been noted by other laboratories. ${ }^{21,22}$ We caution clinical laboratories in the interpretation and reporting of a "negative" Modified Hodge Test. To assure that NDM-1-producing bacteria are detected for epidemiologic purposes and infection control, we recommend that laboratories adopt alternate tests, such as inhibitor-disk analysis comparing susceptibility of suspect organisms to carbapenems with and without carbapenemase inhibitors. ${ }^{13,21}$ Alternatively, laboratories can adopt or work with reference laboratories to provide direct testing of all suspect organisms with polymerase chain reaction.

\section{References}

1. Yong D, Toleman MA, Giske CG, et al. Characterization of a new metallo- $\beta$-lactamase gene, bla(NDM-1), and a novel erythromycin esterase gene carried on a unique genetic structure in Klebsiella pneumoniae sequence type 14 from India. Antimicrob Agents Chemother 2009;53:5046-54.

2. Kumarasamy KK, Toleman MA, Walsh TR, et al. Emergence of a new antibiotic resistance mechanism in India, Pakistan, and the UK: a molecular, biological, and epidemiological study. Lancet Infect Dis 2010;10:597-602.

3. Pillai DR, McGeer A, Low DE. New Delhi metallo- $\beta$-lactamase1 in Enterobacteriaceae: emerging resistance. CMAJ 2011; 183:59-64.

4. Mulvey MR, Grant JM, Plewes K, et al. New Delhi metallo- $\beta$ lactamase in Klebsiella pneumoniae and Escherichia coli, Canada. Emerg Infect Dis 2011;17:103-6.

5. Peirano G, Ahmed-Bentley J, Woodford N, et al. New Delhi metallo- $\beta$-lactamase from traveler returning to Canada. Emerg Infect Dis 2011;17:242-4.

6. Tijet N, Alexander DC, Richardson D, et al. New Delhi metallo$\beta$-lactamase, Ontario, Canada. Emerg Infect Dis 2011;17:306-7.

7. Guidance: Infection prevention and control measures for healthcare workers in all healthcare settings: Carbapenem-resistant Gram-negative bacilli. Ottawa (ON): Public Health Agency of Canada; 2010. Available: www.phac-aspc.gc.ca/nois-sinp /guide/ipcm-mpci/ipcm-mpci-eng.php. (accessed 2011 Mar. 5).

8. Guidance on carbapenem resistance producers. London (UK): UK Health Protection Agency; 2011. Available: www.hpa.org.uk /Topics/InfectiousDiseases/InfectionsAZ/CarbapenemResistance /GuidanceOnCarbapenamProducers/ (accessed 2011 Mar. 5).

9. Carmeli Y, Akova M, Cornaglia G, et al. Controlling the spread of carbapenemase-producing Gram-negatives: therapeutic approach and infection control. Clin Microbiol Infect 2010;16: 102-11.

10. Clinical and Laboratory Standards Institute. Performance standards for antimicrobial susceptibility testing; twenty-first informational supplement. CLSI document M100-S21. Wayne (PA): The Institute; 2011.

11. Detection of Enterobacteriaceae isolates carrying metallo- $\beta$ lactamase - United States, 2010. MMWR Morb Mortal Wkly Rep 2010;59:750.

12. Clinical and Laboratory Standards Institute. Performance standards for antimicrobial susceptibility testing; twentieth informational supplement. CLSI document M100-S20. Wayne (PA): The Institute; 2010.

13. Seah C, Low DE, Patel SN, et al. Comparative evaluation of a chromogenic agar medium, the modified Hodge test, and a battery of meropenem-inhibitor discs for detection of carbapenemase activity in Enterobacteriaceae. J Clin Microbiol 2011;49:1965-9.

15. The European Committee on Antimicrobial Susceptibility Testing (EUCAST) Clinical Breakpoints. Basel (Switzerland): European Society of Clinical Microbiology and Infectious Diseases; 2011. Available: www.eucast.org/clinical_breakpoints (accessed 2011 May 18).

14. Clinical and Laboratory Standards Institute. Methods for dilution antimicrobial susceptibility tests for bacteria that grow aerobically; approved standard - eighth edition. CLSI document M07-A8. Wayne (PA): The Institute; 2009.

16. Product Monograph ${ }^{\mathrm{Pr}}$ Tygacil® tigecycline for injection. Kirkland (QC): Pfizer Canada; 2010. 
17. Murray BE, Singh KV, Heath JD, et al. Comparison of genomic DNAs of different enterococcal isolates using restriction endonucleases with infrequent recognition sites. J Clin Microbiol 1990;28:2059-63.

18. Reekers JA, Lee MJ, Belli AM, et al. Cardiovascular and Interventional Radiological Society of Europe commentary on the treatment of chronic cerebrospinal venous insufficiency. Cardiovasc Intervent Radiol 2011;34:1-2.

19. Nicolle LE, Bradlely S, Colgan R, et al. Infectious Diseases Society of America Guidelines for the diagnosis and treatment of asymptomatic bacteriuria in adults. Clin Infect Dis 2005;40: 643-54.

20. Kus J, Willey BM, Melano R, et al. Failure of the modified Hodge test (MHT) to detect New Delhi metallo- $\beta$-lactamase-1 (NDM-1) carbapenemases. Can J Infect Dis Med Microbiol 2011;22 (Suppl A):41A-42A (Poster No. SP11).

21. Mochon AB, Garner OB, Hindler JA, et al. New Delhi metallo$\beta$-lactamase (NDM-1)-producing Klebsiella penumoniae: case report and laboratory detection strategies. J Clin Microbiol 2011;49:1667-70.

22. Castanheira M, Deshpande LM, Mathai D, et al. Early dissemination of NDM-1- and OXA-181-producing Enterobacteriaceae in Indian hospitals: report from the SENTRY Antimicrobial Surveillance Program, 2006-2007. Antimicrob Agents Chemother 2011;55:1274-8.

Affiliations: From the University Health Network and Mount Sinai Hospital Joseph and Wolf Lebovic Health Complex Department of Microbiology (Kus, Low, McGeer, Willey, Larocque, Pike, Edwards, Dedier, Poutanen) Toronto, Ont.; the University of Toronto (Kus, Tadros, Simor, McGeer, Melano, Bayley, Whitehead, Richardson, Carr, Poutanen) Toronto, Ont.; Sunnybrook Health Sciences Centre (Tadros, Simor, Louie, Carr, Jinnah) Toronto, Ont.; Ontario Agency for Health Protection and Promotion (Low, Melano) Toronto, Ont.; Public Health Agency of Canada (Boyd, Mulvey), Winnipeg, Man.; and the Toronto Rehabilitation Institute (Okeahialam, Bayley, Whitehead, Richardson), Toronto, Ont.

Contributors: Julianne Kus, Manal Tadros, Andrew Simor and Susan Poutanen were responsible for the article's concept. Julianne Kus, Manal Tadros, Andrew Simor, Donald Low, Allison McGeer and Susan Poutanen drafted the manuscript. Julianne Kus, Manal Tadros, Barbara Willey, Cindy Larocque, Karen Pike, Iris Ann Edwards, Helen Dedier, Roberto Melano, David Boyd, Michael Mulvey, Lisa Louie, Andrew Simor, and Susan Poutanen were involved in the detecting the NDM-1-producing organisms and acquiring laboratory data for the manuscript. Christopher Okeahialam, Mark Bayley, Cynthia Whitehead, Denyse Richardson, Julianne Kus, Susan Poutanen, Allison McGeer, Manal Tadros, Lesley Carr and Fatema Jinnah were involved in managing the care of the patients and helped acquire patient data for the manuscript. All of the authors have reviewed, revised and approved the final version of the manuscript submitted for publication.

Funding: No external funding was received for this study.

Acknowledgements: The authors thank Ming Lum and Poolak Akhavan for their help with confirming some of the laboratory data used in this manuscript. 